\title{
Viruses are frequently present as the infecting agent in acute exacerbations of chronic obstructive pulmonary disease in patients presenting to hospital
}

\author{
E. Biancardi, ${ }^{1}$ M. Fennell, ${ }^{2,3}$ W. Rawlinson ${ }^{2,3}$ and P. S. Thomas ${ }^{1,4}$ \\ ${ }^{1}$ Department of Respiratory Medicine, and ${ }^{2}$ Serology and Virology Division (SAViD) SEALS Microbiology, Prince of Wales Hospital, and ${ }^{3}$ School of \\ Medical Sciences and School of Biotechnology and Biomolecular Sciences, The University of New South Wales, and ${ }^{4}$ Prince of Wales Clinical School \\ and Inflammation Infection Research, School of Medical Sciences, University of New South Wales, Sydney, New South Wales, Australia
}

\section{Key words}

acute exacerbation of COPD, respiratory viruses, C-reactive protein, flu-like symptoms, nasopharyngeal aspirate, PCR.

\section{Correspondence}

Edwina Biancardi, Department of Respiratory Medicine, St George Hospital, Gray Street, Kogarah, Sydney, NSW 2217, Australia. Email: edwinabiancardi@gmail.com

Received 11 April 2016; accepted

3 August 2016.

doi:10.1111/imj.13213

\begin{abstract}
Background: Viral causes of acute exacerbations of chronic obstructive pulmonary disease (AECOPD) are well recognised but only recently have rapid tests become available.

Aims: To identify respiratory viruses in the general population and those associated with hospitalisation in AECOPD using polymerase chain reaction (PCR) on nasopharyngeal aspirate (NPA), and the relationship between symptoms, viral detection and inflammatory markers.

Methods: A review of viruses detected in the general population in a health district between August 2014 and July 2015, using multiplex PCR for viruses from NPA samples. In addition, a single hospital, retrospective audit of patients admitted with suspected AECOPD was conducted.

Results: Of the 8811 NPA tested, 5599 (64\%) were positive for at least one virus and 2069 of these were obtained from adults. In adults, the most common viruses identified were Influenza A (31\%), Rhinovirus (27\%) and respiratory syncytial virus A/B (10\%). Most patients with AECOPD (102 of 153) had NPA sent for viral PCR testing and $59(58 \%)$ were positive. The most common viruses identified were Influenza A $(31 \%)$, Rhinovirus (24\%) and respiratory syncytial virus A/B (17\%) with co-infecting bacteria cultured in 22 sputum samples. Patients with influenza-like symptoms were more likely to have a positive viral PCR than those without symptoms $(P<0.004)$. The median Creactive protein on admission was lower in the virus-infected than uninfected AECOPD (28 vs $60 \mathrm{mg} / \mathrm{L}, P<0.026)$.

Conclusion: The spectrum of viruses detected in patients with AECOPD is similar to that of the general population. Viruses are more likely to be identified in patients with AECOPD who present with influenza-like symptoms and a low C-reactive protein.
\end{abstract}

\section{Introduction}

Chronic obstructive pulmonary disease (COPD) constitutes a major health problem in Australia and acute exacerbations of COPD (AECOPD) have considerable impact on morbidity, mortality and quality of life. ${ }^{1}$ Common triggers for AECOPD include air pollution and infection with viruses and/or bacteria in the tracheobronchial tree. However, in at least one-third of more severe exacerbations a cause is not identified. Antibiotic therapy is widely, and sometimes inappropriately, used in AECOPD resulting in increased risk of adverse effects and increased

Funding: None.

Conflict of interest: None. antibiotic resistance. Antiviral therapy for influenza viruses is available and needs to be commenced soon after the development of symptoms. Antiviral agents for other viruses including anti-rhinoviral agents are in clinical development, and not yet available for routine use.

Previous longitudinal, community-based and hospitalisation studies showed viral infections are common $(39-56 \%)$ in patients with AECOPD, and are most usually associated with severe presentations, ${ }^{2}$ and with a longer median symptom recovery compared with non-viral exacerbations. ${ }^{2}$ The viruses detected in the upper respiratory tract most frequently are picornaviruses such as human rhinovirus or hRV (36\%), influenza A $(25 \%)$ and respiratory syncytial virus (RSV) $(22 \%))^{3-5}$ 
While viral causes of AECOPD are well recognised, sensitive and rapid tests for common respiratory viruses have only recently been used widely in clinical settings. Comparison of different diagnostic methods for the detection of respiratory viruses including viral culture, antigen detection tests, serology and reverse transcriptase PCR have shown that nucleic acid tests (NAT) such as PCR are more sensitive and at least equally specific. ${ }^{3}$ The PCR technique using nasopharyngeal aspirates (NPA) or swabs has a 2.7 times higher diagnostic yield ${ }^{6}$ than conventional viral culture and thus has been widely used in the South Eastern Sydney Laboratory Services (SEALS) reference virology laboratories in the Prince of Wales Hospital (POWH) over the past 2 years, as the screening tool for viral respiratory tract infections.

Given the time between NPA sampling, NAT assay and availability of results, alternate sensitive, but potentially less specific markers that differentiate viral and bacterial respiratory infections would be beneficial to guide initial therapy. C-reactive protein (CRP) is an acute phase protein that increases with infectious and inflammatory conditions. CRP levels have been shown to increase in AECOPD but are not well correlated to severity or outcome. ${ }^{7}$ Few studies have examined the relationship between microbial aetiology and CRP levels in patients with AECOPD.

Within POWH, a single centre in the Sydney Eastern Suburbs Local Health District (SESLHD), most respiratory patients presenting to the Emergency Department (ED) who are likely to require admission and who have symptoms suggestive of a viral respiratory tract infection during the annual influenza season undergo NPA for viral PCR and isolation until the results are available. In this group of patients, a significant proportion has underlying COPD and these patients are commenced on antimicrobial treatment based on clinical criteria for AECOPD. It would be helpful to have early indicators of viral infection, either by direct identification of viruses or by a surrogate. This would allow appropriate treatment and isolation of such patients. The aim of this study was to assess the respiratory viruses associated with hospitalisation in AECOPD identified by NPA viral PCR testing and the relationship between viral detection, inflammatory markers and co-existent organisms.

\section{Methods}

This was a retrospective case study design that was approved by the SESLHD Ethics Committee. Two audits were conducted in this study over the same time period:

(1) The details of all NPA requested from a general population within one local health district;

(2) A specific group of patients admitted to a single hospital in the same local health district.
(1) An audit was performed upon the database of all NPA submitted for viral PCR in the SESLHD to the Department of Virology and Serology (SAViD) SEALS Microbiology between 1 August 2014 and 31 July 2015. The subjects were of all age groups and tests were requested from primary healthcare, outpatient and inpatient settings. Baseline characteristics, co-morbidities and indications for testing were not available for this cohort.

(2) A separate detailed audit was performed of the medical records of all patients admitted with an AECOPD during the period between 1 October 2014 and 31 September 2015 under a respiratory physician at POWH in Sydney, who met the inclusion criteria. Inclusion criteria were: (i) age $>18$ years (ii) previous diagnosis of COPD or Spirometry performed on admission revealing $\mathrm{FEV}_{1} / \mathrm{FVC}<70 \%$ and (iii) symptoms of AECOPD as per the GOLD definition (worsening of the patient's respiratory symptoms that is beyond normal day-today variations and leads to a change in medication). ${ }^{8}$ The GOLD definition of AECOPD refers to an acute change in one or more of the following cardinal symptoms: an increase in cough frequency/severity, an increase in sputum volume/ change in character and an increase in dyspnoea.

The severity of COPD was defined by the GOLD criteria, that is, mild: $\mathrm{FEV}_{1} \geq 80 \%$ predicted, moderate: $\mathrm{FEV}_{1}$ $50-79 \%$, severe $30-49 \%$ and very severe $\mathrm{FEV}_{1}<30 \%{ }^{8}$ The audit was based on the definition of influenza-like symptoms on the local guidelines for viral NPA testing followed in the ED: patients presenting with fever $>38^{\circ} \mathrm{C}$ plus one or more of coryzal symptoms, myalgia or headache.

The electronic pathology database was searched for results of viral NPA PCR, CRP or sputum microscopy and culture specimens requested within the first $24 \mathrm{~h}$ of arrival to hospital. Similarly, chest x-ray reports from presentation were reviewed and the presence of parenchymal collapse/consolidation noted. The commencement of empirical antibiotics or antiviral agents in the ED was recorded if documentation confirmed their indication was for the treatment of an AECOPD. Instructions to isolate patients with droplet precautions from admission were recorded if documented by emergency staff.

\section{Results}

\section{Patient selection}

\section{General population}

The SESLHD virology database containing 8811 NPA viral PCR results was interrogated and showed 5599 $(64 \%)$ positive results in the year between August 2014 and July 2015 (Fig. 1.) Of the 5599 positive results, 2069 of these NPA were obtained from adults and were positive for at least one of the viruses graphed in Figure 2. 

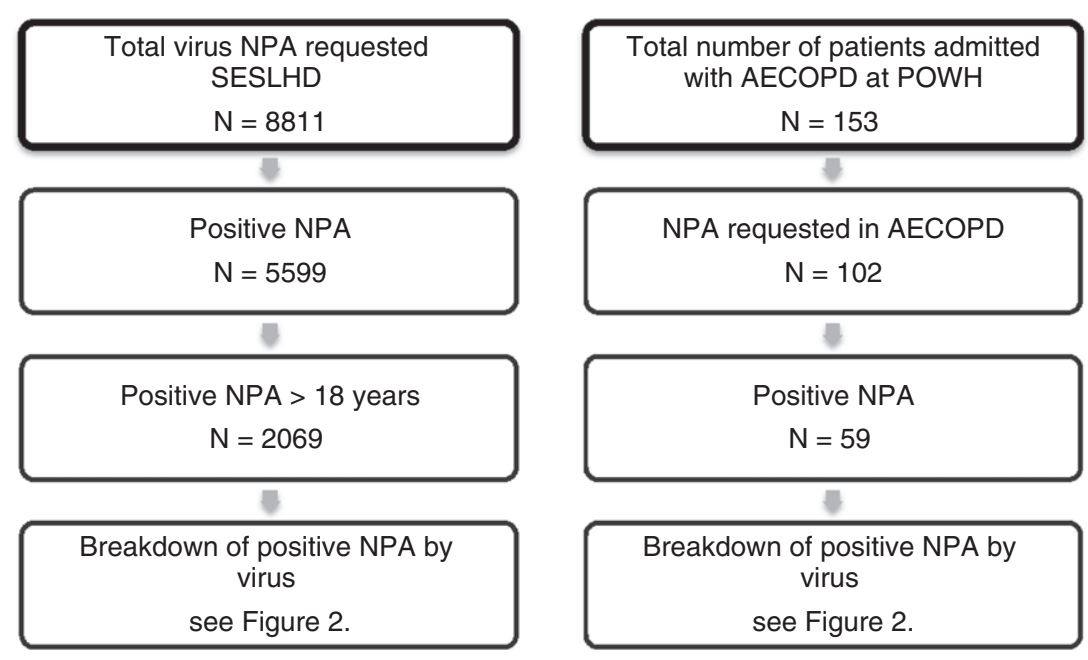

Figure 1 Uptake of NPA respiratory virus testing by PCR over 12 months. AECOPD, acute exacerbations of chronic obstructive pulmonary disease; NPA, nasopharyngeal aspirate; $\mathrm{PCR}$, polymerase chain reaction; POWH, Prince of Wales Hospital; SESLHD, Sydney Eastern Suburbs Local Health District.
The most common viruses identified in this adult, allcomer population were Influenza A 31\% (642), Rhinovirus $27 \%$ (565) and RSV A/B 10\% (209) (Fig. 2). The profile of viruses from the 3503 patients less than 18 years of age was significantly different to the adult group.

\section{Patients with AECOPD}

Medical records from a single centre within the SESLHD, POWH found that 153 patients were admitted with AECOPD in a similar 12-month period. The clincial characteristics of the patients are shown in Table 1. The cohort had a mean age of 72 years (range: 32-98). Prior to the index presentation, COPD was previously recognised in $130(85 \%)$ patients and of those in whom spirometry was initially performed, the majority were classified as having severe COPD. The remaining 23 (15\%) patients were found to have obstructive spirometry on presentation consistent with COPD; however, spirometric data to exclude significant bronchial reversibility were not available so that asthma could not be excluded.

\section{Virus identification}

NPA $(n=102)$ were sent for viral PCR of which $59(55 \%)$ were positive.

The most common viruses identified were Influenza A $31 \%$ (18), Rhinovirus 24\% (14) and RSV A/B 17\% (10) (Fig. 2). Multiple viruses were identified in three patients with positive viral PCR. These co-infections included Influenza B/Rhinovirus/Coronavirus, RSV/Coronavirus and Rhinovirus/Coronavirus.

In those with a positive NPA, $13(22 \%)$ cultured Coexistent bacteria in sputum tested within $24 \mathrm{~h}$ of admission. The most common bacteria and fungi cultured included Pseudomonas aeruginosa (31\%), Haemophilus influenzae (23\%) and Aspergillus species (23\%).

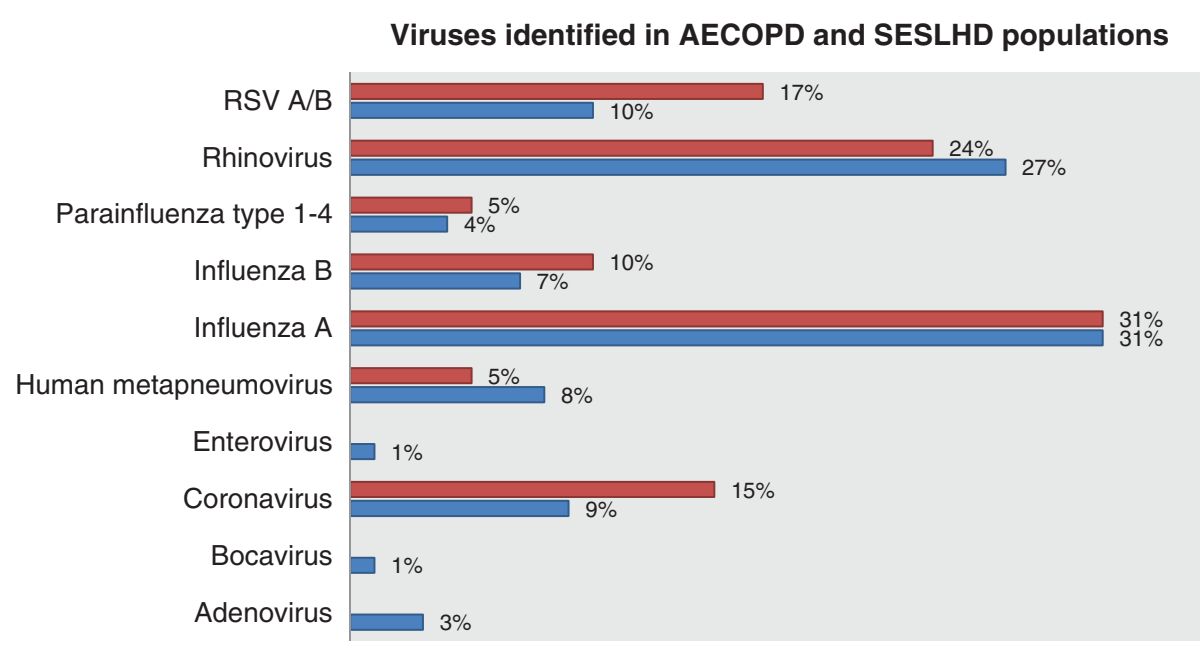

Figure 2 Percentage of viruses identified in AECOPD and SESLHD populations. ( $\square$ ), AECOPD, acute exacerbations of chronic obstructive pulmonary disease; RSV, respiratory syncytial virus; $\square$, SESLHD, Sydney Eastern Suburbs Local Health District. 
Table 1 Clinical characteristics of patients presenting with AECOPD

\begin{tabular}{lc}
\hline Characteristics & Number (\%) \\
\hline Gender & \\
Male & $77(50)$ \\
Female & $76(50)$ \\
Baseline COPD severity & \\
$\quad$ Mild & $5(3)$ \\
Moderate & $16(10)$ \\
Severe & $43(28)$ \\
Very severe & $29(19)$ \\
Unknown & $60(39)$ \\
Influenza-like symptoms & \\
Yes & $75(49)$ \\
No & $78(51)$ \\
CXR consolidation & \\
Yes & $53(35)$ \\
No & $100(65)$ \\
Viral NPA requested & \\
Yes & $102(67)$ \\
No & $51(33)$ \\
Inflammatory markers requested & \\
CRP & $94(61)$ \\
Pro-calcitonin & $6(6)$ \\
Empirical antibiotics commenced & \\
Yes & $151(99)$ \\
No & $2(1)$ \\
Empirical antivirals commenced & \\
Yes & $15(10)$ \\
No & $138(90)$ \\
Yes & \\
No & $45(29)$ \\
\hline AECOPD & $108(71)$ \\
\hline
\end{tabular}

AECOPD, acute exacerbations of chronic obstructive pulmonary disease; COPD, Chronic obstructive pulmonary disease; CRP, C-reactive protein; CXR; NPA, nasopharyngeal aspirate.

\section{Clinical associations}

\section{Symptoms}

Influenza-like symptoms (fever plus one or more of coryzal symptoms, myalgia or headache) were reported by $66(65 \%)$ of 102 patients on presentation to the ED who underwent viral NPA PCR testing (Fig. 3).

Those with influenza-like symptoms were statistically more likely to have a positive viral NPA PCR than those without symptoms ( $P$ value $<0.004$, Chi-squared test).

\section{Severity of COPD}

The severity of COPD was able to be estimated for $60 \%$ of all patients and $83 \%$ of those with positive viral PCR. The majority of patients admitted met GOLD criteria for severe and very severe COPD, but this did not relate to the likelihood of viral positivity.

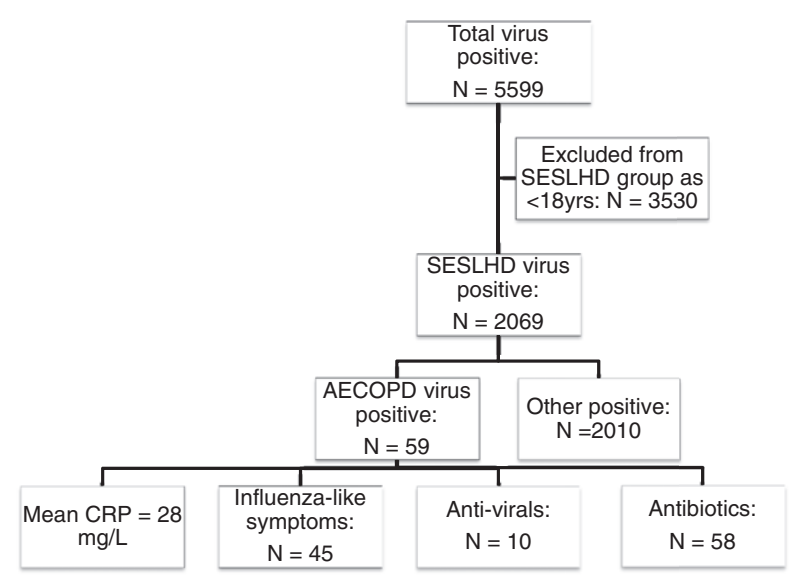

Figure 3 Relationship between virus positive NPA in SESLHD and AECOPD cohorts. AECOPD, acute exacerbations of chronic obstructive pulmonary disease; CRP, C-reactive protein; NPA, nasopharyngeal aspirate; SESLHD, Sydney Eastern Suburbs Local Health District.

\section{Inflammatory markers}

CRP was requested in approximately two-thirds of patients presenting to the ED with AECOPD. In those with a positive viral PCR, the mean CRP was highest in those with RSV A/B (140 mg/L (16 419)) followed by Rhinovirus (69 mg/L (3340)) and Influenza A (52 mg/L (9268)). Median CRP on admission was lower in the patients with a viral versus non-viral AECOPD $(28 \mathrm{mg} / \mathrm{L}$ (1419) vs $60 \mathrm{mg} / \mathrm{L}$ (6296) with $P$ value <0.02, MannWhitney).

\section{Radiology}

The radiology was reviewed and evidence of consolidation was found in 19 of those with a positive viral NPA PCR, and 17 of those which were negative.

\section{Seasonal variation in viral detection}

The seasonal trend in positive viral PCR results in the SESLHD database reflected the peak 4 weeks (4 August 2014-31 August 2014) of laboratory notifications of influenza in NSW during the $2014 \mathrm{flu}$ season. ${ }^{9}$ Of the 1034 positive influenza PCR results between 1 August 2014 and 31 July 2015, 43\% (443) were confirmed in the month of August alone.

\section{Isolation}

Based on initial clinical history, examination and radiology findings, 45 (29\%) patients with AECOPD were isolated with droplet precautions as per the influenza season protocol in the ED. Of those 45 patients, $33(73 \%)$ were found to have a positive viral PCR. In 
the group of 108 patients who were not initially isolated, $26(24 \%)$ were found to have a positive viral PCR.

\section{Empirical antimicrobials}

Of the $151(99 \%)$ patients commenced on empirical antibiotics for AECOPD, 58 (38\%) had a positive viral PCR. A minority of patients, (10 (67\%) of 15 patients) were commenced on empirical Oseltamivir and had a positive viral PCR.

\section{Discussion}

This study retrospectively examined the relationship between the presenting symptoms, inflammatory markers and microbiological results of patients presenting to a single centre with AECOPD after the introduction of viral NPA PCR testing. It demonstrates that the detection rate of respiratory viruses was strongly associated with a low CRP level and influenza-like symptoms on presentation.

When comparing the POWH AECOPD data to that of the wider health district (SESLHD) over a similar time period, some interesting observations can be drawn. The microbiological profile of viruses identified between the adults groups was similar, revealing Influenza A to be the most common virus isolated regardless of underlying COPD followed by rhinovirus, RSV and coronaviridae. It would be informative to establish the proportion of patients with COPD in the POWH group that had received the annual influenza vaccination prior to their presentation, but this information was rarely recorded in the medical record. Compared to the general population captured in the SESLHD data, one would expect this at risk group to have more protection against influenza through greater vaccination rates.

Viral and bacterial infections trigger AECOPD in up to $80 \%$ of cases. ${ }^{10}$ Co-infection with bacteria and viruses, as in $22 \%$ of our patients, suggests that both classes of organisms play a role. In studies of asthma exacerbations, it appears likely that respiratory viruses are the principal initiating infections, with initial bacterial infections being of lesser importance. While the reason behind lower rates of viral detection in exacerbations of COPD compared to asthma is unclear, the overall detection rate of $55 \%$ in this study is slightly higher than other similar studies. ${ }^{2,3}$ It would appear likely that viral infections disrupt the airway epithelium, reducing the innate immunity and allowing bacterial secondary infection to occur, as has been previously described. ${ }^{11}$

Surrogate markers to discriminate viral and non-viral AECOPD were reviewed in this audit and both symptoms and inflammatory markers were found to be potentially useful indicators. The current guidelines regarding influenza-like symptoms was found to be strongly associated with positive viral results and thus appear to support this clinical assessment as a key aspect of the history obtained on presentation for triage. Further studies associating influenza-like symptoms and rates of droplet precaution isolation with proven viral infection would determine the necessity of an isolation protocol for such patients and be of public health interest to prevent in-hospital spread.

Previous studies of AECOPD have shown that in patients who have increased sputum purulence, the pattern of increase in CRP is similar to that seen in patients with pneumonia. ${ }^{12}$ This suggests that CRP may be used as a marker of significant bacterial infection and may have a role in determining whether antimicrobials should be prescribed. CRP levels were significantly lower in patients with viral AECOPD, supporting the contributory role of this serum marker in establishing the aetiology of AECOPD and its potential to guide treatment.

In previous COPD studies, co-infection appears to increase the severity of the exacerbation, and is associated with more symptoms, higher bacterial loads and systemic inflammation. ${ }^{13}$ These findings have implications for treatment. While newly acquired viruses may be the initiating agent, one study suggests antibiotics may minimise the interaction of chronic, colonising bacteria with virus that would have resulted in an increase in bacterial load or phenotypic change. ${ }^{7}$ However, this argument needs to be weighed against the potential for empirical antibiotics to cause antimicrobial resistance in an already vulnerable population. This audit has found symptoms and markers of inflammation to be informative in identifying a cause of AECOPD.

Owing to the study design, there are limitations in attributing causation of viruses detected to AECOPD. First, viral infection may have been missed in up to onethird of patients who were not tested on presentation, presumably due to lack of influenza-like symptoms. As viral shedding can occur for several weeks after postinfection, routine testing of all subjects may have affected the study's findings including the associations of symptoms and inflammatory markers. Second, potential selection bias relating to the indication for viral testing exists between the two cohorts limiting the capacity to compare data sets. The AECOPD cohort was a highly selected group of patients, many of whom had symptoms suggestive of a viral infection to prompt testing. In comparison, the indication for testing in the SESLHD cohort is unknown and likely to be variable, including some screening for infection control. In the future, prospective studies could address whether CRP, and perhaps pro-calcitonin, could be used in addition to clinical acumen to guide empirical antimicrobial use in AECOPD. Likewise, large controlled trials of patients randomised to 
receive empirical antimicrobials or targeted viral therapy alone in suspected viral AECOPD may further identify subgroups which would benefit most from antimicrobial treatment.

\section{Conclusions}

This audit found the spectrum of viruses detected in the upper respiratory tract of patients presenting with AECOPD to be similar to that of a general, adult population. Amongst the AECOPD cohort, viruses appear more likely to be identified in those who present with influenza-like symptoms and a low CRP. These findings support the potential role of symptoms and inflammatory markers in determining the aetiology and guiding empirical management of AECOPD. Given the prevalence of Influenza A in the AECOPD cohort, widespread vaccination may help to protect these patients and prevent hospital admission.

\section{References}

1 Daniels JMA, Schoorl M, Snijders D, Know DL, Lutter R, Jansen HM et al. Procalcitonin vs. C-reactive protein as predictive markers of response to antibiotic therapy in acute exacerbations of COPD. Chest 2010; 138: 1108-5.

2 Seemungal T, Harper-Owen R, Bhowmik A, Moric I, Sanderson G, Message $S$ et al. Respiratory viruses, symptoms, and inflammatory markers in acute exacerbations and stable chronic obstructive pulmonary disease. Am J Respir Crit Care Med 2001; 164: 1618-23.

3 Rohde G, Wiethege A, Borg I, Kauth M, Bauer TT, Gillissen A et al. Respiratory viruses in exacerbations of chronic obstructive pulmonary disease requiring hospitalisation: a case-control study. Thorax 2003; 58: 37-42.

4 Walsh EE, Falsey AR, Hennessey PA. Respiratory syncytial and other virus infections in persons with chronic cardiopulmonary disease.
Am J Respir Crit Care Med 1999; 160: 791-5.

5 Greenberg SB, Allen M, Wilson J, Atmar RL. Respiratory viral infections in adults with and without chronic obstructive pulmonary disease. Am J Respir Crit Care Med 2000; 162: 167-73.

6 Ko FW, Ip M, Chan PK, Chan MC, To KW, Ng SS et al. Viral etiology of acute exacerbations of chronic obstructive pulmonary disease in Hong Kong. Chest 2007; 132 900.

7 Clark TW, Medina MJ, Batham S, Curran MD, Parmar S, Nicholson KG. C-reactive protein level and microbial aetiology in patients hospitalised with acute exacerbation of COPD. Eur Respir J 2015; 45: 76-86.

8 Global Initiative for Chronic Obstructive Lung Disease. [cited 2016 Sep 10] Available from URL: http://goldcopd. org/gold-spirometry-guide/

9 Dalton CB, Carlson SJ, McCallum L, Butler MT, Elvidge E, Durrheim DN.
Flutracking weekly online community survey of influenza-like illness: 2013 and 2014. Commun Dis Intell Q Rep 2015; 39: 361-8.

10 Sapey E, Stockley RA. COPD exacerbations 2: aetiology. Thorax 2006; 61: $250-8$

11 Jamieson AM, Yu S, Annicelli CH, Medzhitov R. Influenza virus-induced glucocorticoids compromise innate host defense against a secondary bacterial infection. Cell Host Microbe 2010; 7 : 103-4.

12 Weis N, Almdal T. C-reactive protein can it be used as a marker of infection in patients with exacerbation of chronic obstructive pulmonary disease? Eur J Intern Med 2006; 17: 88-91.

13 Wilkinson TM, Hurst JR, Perera WR, Wilks M, Donalsdon GC, Wedzicha JA. Effect of interactions between lower airway bacterial and rhinoviral infection in exacerbations of COPD. Chest 2006; 129: 317-24. 